13.4

\title{
Влияние рассеянных электронов на коэффициент шума циклотронных защитных устройств
}

\author{
(C) С.В. Быковский \\ НПП „Исток“ им. А.И. Шокина, Фрязино, Московская обл., Россия \\ E-mail: serbyko@mail.ru
}

Поступило в Редакцию 14 января 2021 г.

В окончательной редакции 26 марта 2021 г.

Принято к публикации 29 марта 2021 г.

\begin{abstract}
Экспериментально исследовано влияние рассеянных электронов луча на шумовые параметры циклотронных защитных устройств сверхвысокочастотного диапазона. Показано, что электроны луча, рассеянные на частицах остаточных газов, являются источником дополнительных шумов устройства. В качестве объекта исследований использовались серийно выпускаемые образцы циклотронных защитных устройств.
\end{abstract}

Ключевые слова: циклотронное защитное устройство, ленточный электронный луч, быстрая циклотронная волна, коэффициент шума.

DOI: 10.21883/PJTF.2021.12.51069.18711

Одним из путей совершенствования параметров современных радиолокационных систем является использование циклотронных защитных устройств (ЦЗУ) для защиты входных каскадов приемников от мощных сверхвысокочастотных (СВЧ) импульсов. ЦЗУ отличает сверхмалое время восстановления параметров (10-20 ns) после окончания мощного СВЧ-импульса, высокое качество защиты, низкий коэффициент шума $(0.7-1.2 \mathrm{~dB})$ в режиме пропускания сигнала [1,2]. Цель настоящей работы - исследовать влияние ухудшения вакуума в устройстве вследствие газоотделения или воздействия внешних факторов на коэффициент шума.

ЦЗУ - это вакуумный электронно-лучевой прибор, в котором передача сигнала с его входа на выход происходит посредством возбуждения в луче быстрой циклотронной волны во входном резонаторе и выведения ее энергии в выходном резонаторе. Передача сигнала линейна по мощности до уровня нескольких $\mathrm{mW}$, при большей входной мощности электронный луч разрушается и устройство переходит в режим защиты.

Для обеспечения минимально возможного коэффициента шума ЦЗУ следует минимизировать рассеяние электронов луча на ионах, атомах и молекулах остаточных газов, присутствующих в вакуумном объеме устройства. Рабочий уровень вакуума в ЦЗУ составляет $\sim 10^{-7}-10^{-8} \mathrm{~mm} \mathrm{Hg}$, при ухудшении вакуума относительно указанного уровня в области электронного луча образуется значительное количество рассеянных электронов. Их колебания с частотой, близкой к циклотронной, наводят в выходном резонаторе шумовые токи и, таким образом, являются источником собственной шумовой мощности ЦЗУ.

Рассматривая влияние рассеянных электронов на коэффициент шума ЦЗУ, следует выделить два основных аспекта: диаметр вращательных колебаний электронов в продольном магнитном поле устройства и частоту колебаний.

Диаметр циклотронного вращения рассеянных электронов важен в том смысле, что если он близок к величине электронного зазора $d$ (рис. 1) в резонаторах ЦЗУ или превосходит ее, то такие электроны будут практически сразу оседать на ламелях и не будут оказывать заметного влияния на коэффициент шума. Электроны, рассеянные под малыми углами относительно продольной оси луча, будут достаточно быстро уходить из резонатора и также не окажут существенного влияния на коэффициент шума. Однако рассеянные электроны c амплитудой циклотронных колебаний существенно меньше поперечного размера электронного зазора $d$, потерявшие продольную скорость, могут достаточно долго существовать в объеме электронного луча и наводить шумовые токи в выходном резонаторе.

Оценим радиус $R$ поперечного циклотронного вращения рассеянных электронов в предположении упругого столкновения с частицами остаточного газа с направлением движения после соударения перпендикулярно продольному магнитному полю. При этом электрон будет совершать циклотронное вращение с частотой $\omega_{c}$ (без учета влияния поля пространственного заряда). Будем предполагать, что ЦЗУ работает с предельно малыми мощностями на входе, при которых собственно и важен низкий коэффициент шума. В этом случае энергия электрона до столкновения определяется потенциалом дрейфа луча $U_{0}$, после столкновения энергия переходит в энергию вращения с циклотронной частотой $\omega_{c}$ и радиусом $R=\sqrt{2 \eta U_{0}} / \omega_{c}$, где $\eta=e / m$ - отношение заряда электрона к его массе.

При типичном потенциале дрейфа $U_{0}=20 \mathrm{~V}$ и угле рассеяния электронов 45-90 ${ }^{\circ}$ относительно продольной оси луча диаметр циклотронного вращения наиболее существенной доли рассеянных электронов для частоты 


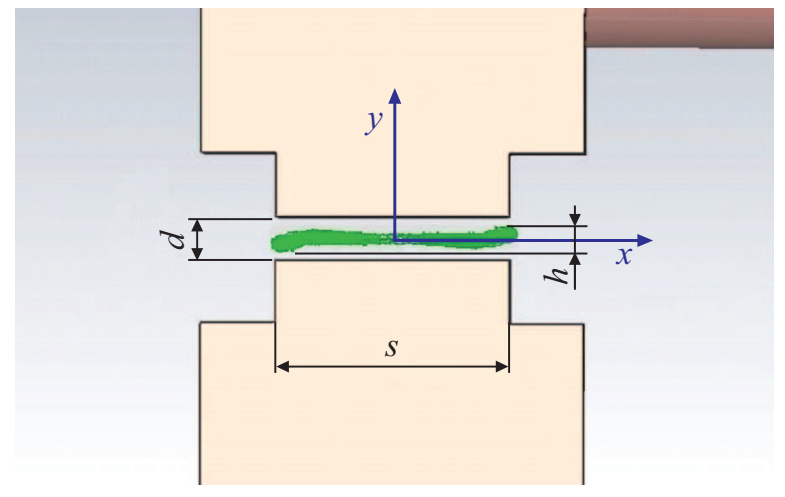

Рис. 1. Поперечное сечение ленточного электронного луча в зазоре $d$ между ламелями резонатора $(s-$ ширина рабочей части зазора, $h$ - толщина луча с учетом деформации его поперечного сечения в продольном магнитном поле).

$3 \mathrm{GHz}$ приблизительно составит $200-280 \mu \mathrm{m}$, а для частоты $9 \mathrm{GHz}-65-100 \mu \mathrm{m}$.

Таким образом, для ЦЗУ диапазона частот $3 \mathrm{GHz}$ с типичным поперечным размером зазора резонатора $d=200-230 \mu \mathrm{m}$ и потенциалом дрейфа луча $U_{0} \geqslant 20 \mathrm{~V}$ рассеянные электроны не способны совершать колебания в зазоре резонатора, так как диаметр их циклотронного вращения приблизительно равен или превышает поперечный размер зазора резонатора $d$.

В ЦЗУ диапазона частот $9 \mathrm{GHz}$ и в более высокочастотных ЦЗУ с зазором $d=130-140 \mu \mathrm{m}$, потенциалом дрейфа $U_{0} \leqslant 20 \mathrm{~V}$ рассеянные электроны являются стабильным источником шума в форме шумовых „вакуумных пиков“ на частотах их колебаний.

Частоты колебаний электронов внутри луча $\omega_{c}^{\prime}$ и $\omega_{c}^{\prime \prime}$ в условиях влияния поля пространственного заряда определяются выражениями $[3,4]$ :

$$
\begin{aligned}
& \omega_{c}^{\prime}=\frac{\omega_{c}}{2}\left(1-\sqrt{1-2\left(\frac{\omega_{p}}{\omega_{c}}\right)^{2}}\right), \\
& \omega_{c}^{\prime \prime}=\frac{\omega_{c}}{2}\left(1+\sqrt{1-2\left(\frac{\omega_{p}}{\omega_{c}}\right)^{2}}\right),
\end{aligned}
$$

где плазменная частота $\omega_{p}=\sqrt{\eta \rho_{e} / \varepsilon_{0}}, \rho_{e}$ - плотность пространственного заряда луча, $\varepsilon_{0}-$ электрическая постоянная. Частоты $\omega_{c}^{\prime}$ и $\omega_{c}^{\prime \prime}$ характеризуют движение электронов по эпициклоидам: $\omega_{c}^{\prime}-$ медленный поворот локальной орбиты вращения электрона и вращение круглого луча как целого (вращение фрагмента ленточного луча), $\omega_{c}^{\prime \prime}$ - быстрое внутреннее вращение электрона.

Выражения (1) получены для электронного луча круглого поперечного сечения. Для ленточного луча, используемого в ЦЗУ, подобные выражения не могут быть получены относительно простым аналитическим способом. При этом они остаются справедливыми для случая однородного или монотонно изменяющегося магнитного поля аксиально-симметричной структуры, которая имеет место в ЦЗУ. Плоскосимметричная структура электрического поля пространственного заряда „усредняется“ за один циклотронный оборот электрона и не оказывает вляния на частоту колебаний [5]. Таким образом, представляется допустимым провести оценки частоты $\omega_{c}^{\prime \prime}$ для типичных плотностей пространствнного заряда луча ЦЗУ и поверить экспериментально указанное допущение. Частоты $\omega_{c}^{\prime}$ отстоят довольно далеко от рабочей полосы частот устройства и не могут регистрироваться посредством измерения коэффициента шума.

Выполним оценки частоты $\omega_{c}^{\prime \prime}$. При условно „низкой““ плотности пространственного заряда луча ЦЗУ частота $\omega_{c}^{\prime \prime}$ незначительно отличается от циклотронной частоты $\omega_{c}\left(\omega_{c}\right.$ соответствует средней частоте рабочей полосы частот ЦЗУ $\left.f_{0}=\omega_{c} / 2 \pi\right)$. При повышенной плотности пространственного заряда (ток луча $250 \mu \mathrm{A}$, потенциал дрейфа $U_{0}=12 \mathrm{~V}$, поперечное сечение луча $0.02 \times 0.7 \mathrm{~mm})$ частота $f_{c}^{\prime \prime}=\omega_{c}^{\prime \prime} / 2 \pi$ не попадет в рабочую полосу частот низкочастотных ЦЗУ (для частоты $f_{0}=3 \mathrm{GHz}$ в приведенных условиях отстоит более чем на $40 \%$ от $f_{0}$ ). Для высокочастотных ЦЗУ $f_{c}^{\prime \prime}$ существенно ближе к центральной частоте устройства (отстоит приблизительно на $2.8 \%$ от $f_{0}=9 \mathrm{GHz}$ ), находится в рабочей полосе частот. Колебания рассеянных электронов при достаточном их количестве формируют на частоте $f_{c}^{\prime \prime}$ пики шумовой энергии.

На экспериментальных кривых (рис. 2) измеренного коэффициента шума ЦЗУ диапазона частот $9 \mathrm{GHz}$ с подключенным к его выходу малошумящим усилителем (МШУ) (с коэффициентом шума $N F=1.0 \mathrm{~dB}$ ) приведены соответствующие частотные зависимости. Ухудшение уровня вакуума в ЦЗУ, сопровождающееся образованием шумового „вакуумного пика“, достигалось путем кратковременного (10-15 s) локального нагрева одного из элементов вакуумной оболочки устройства до температуры приблизительно $200^{\circ} \mathrm{C}$ при помощи газовой горелки. Более продолжительный нагрев приводит к увеличению „вакуумного пика“ по амплитуде и расширению его в полосе частот. Инструментальное измерение изменения уровня вакуума невозможно в рамках существующей технологии.

ЦЗУ, характеристики которого приведены на рис. 2, имеет следующий электрический режим: ток луча $200 \mu \mathrm{A}$, потенциал дрейфа $U_{0}=12 \mathrm{~V}$, поперечное сечение луча ориентировочно $0.04 \times 1.2 \mathrm{~mm}$. Таким образом, аналитическая оценка частоты шумового „вакуумного пика“" дает значение $f_{c}^{\prime \prime}=f_{0}-0.65 \%$. В эксперименте (рис. $2, b)$ шумовой пик смещен на частоту $f_{0}-0.6 \%$. Ошибка и погрешности методики очевидно связаны с приблизительной оценкой поперечного сечения электронного луча.

Кривые на рис. 2, $c$ и $d$ демонстрируют смещение шумового пика по частоте при изменении плотности пространственного заряда луча путем изменения потенциала дрейфа $U_{0}$ : рис. $2, c-$ снижение потенциала до $U_{0}=10 \mathrm{~V}$, увеличение плотности заряда луча, смещение шумового пика по частоте вниз (маркер 4); 

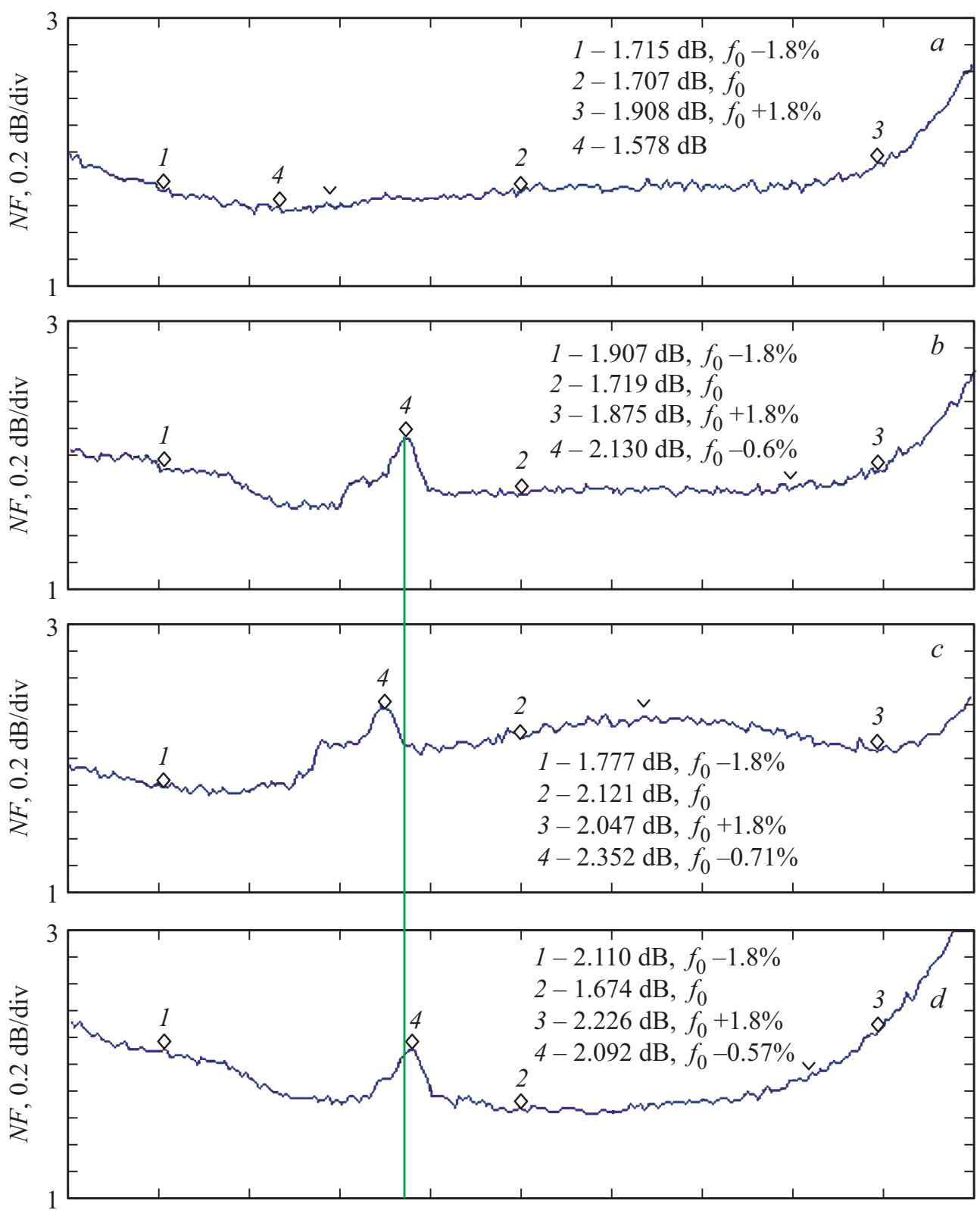

Рис. 2. Частотные зависимости коэффициента шума ЦЗУ+МШУ диапазона частот $9 \mathrm{GHz}(N F=1.0 \mathrm{~dB}) . a-6 е з$ „вакуумного пика“ рассеянных электронов, $b-$ „вакуумный пик“ (маркер 4) при том же режиме электронного луча, $c-$ „вакуумный пик“ при увеличенной плотности объемного заряда луча, $d-$ „вакуумный пик“ при пониженной плотности объемного заряда луча.

рис. $2, d$ - повышение потенциала до $U_{0}=14 \mathrm{~V}$, уменьшение плотности заряда луча, смещение шумового пика по частоте вверх (маркер 4).

Ухудшение коэффициента шума на частотах в стороне от шумовых пиков (кривые на рис. 2, $c$ и $d$ ) связано с неоптимальным электрическим режимом ЦЗУ по потенциалу дрейфа $U_{0}$ в сравнении с кривыми на рис. $2, a$ и $b$.

В заключение следует отметить, что выражение (1) для $\omega_{c}^{\prime \prime}$ позволяет вполне адекватно оценить частоту внутренних колебаний электронов в ленточном луче.

Для практической реализации минимально возможных значений коэффициента шума ЦЗУ крайне важным является высокий уровень вакуума в приборе. Рас сеяние электронов луча на частицах остаточных газов при недостаточном уровне вакуума и плохом обезгаживании внутренней поверхности вакуумной оболочки может значительно ухудшать коэффициент шума, в том числе при повышенной рабочей температуре и других внешних воздействующих факторах. При этом конструктивные особенности, электрический режим и диапазон рабочих частот существенно влияют на чувствительность коэффициента шума ЦЗУ к уровню вакуума в приборе.

Представленные экспериментальные результаты могут быть полезны при исследовании различных процессов в нерелятивистских электронных пучках. 


\section{Благодарности}

Автор выражает благодарность Ю.А. Будзинскому за полезные обсуждения.

\section{Конфликт интересов}

Автор заявляет, что у него нет конфликта интересов.

\section{Список литературы}

[1] С.В. Быковский, Ю.А. Будзинский, В.Г. Калина, В.Е.Котов, О.А. Саврухин, Электронная техника. Сер. 1. СВЧ-техника, B. 3, 43 (2018).

http://www.istokmw.ru/uploads/files/static/101/UHF2018-3.pdf

[2] С.В. Быковский, Ю.А. Будзинский, В.Г. Калина, В.Е. Котов, С.В. Николаев, О.А. Саврухин, Электронная техника. Сер. 1. СВЧ-техника, В. 1, 12 (2020).

http://www.istokmw.ru/uploads/files/static/101/UHF2020-1.pdf

[3] R. Adler, A. Ashkin, E.I. Gordon, J. Appl. Phys., 32 (4), 672 (1961). https://doi.org/10.1063/1.1736068

[4] В.М. Лопухин, А.С. Рошаль, Электронно-лучевые параметрические усилители (Сов. радио, М., 1968). с. 135.

[5] С.В. Быковский, В.А. Ванке, Радиотехника и электроника, 38 (8), 1475 (1993). 